\title{
Lessons learned from using CDK 4/6 inhibitors to treat metastatic breast cancer
}

\author{
Jame Abraham, MD, FACP
}

I

$t$ is amazing to see how many new drugs are being developed and approved for patients with cancer. In 2015 alone, the US Food and Drug Administration approved 45 new cancer drugs - a significant jump from the average 26 approvals annually from 2006 to 2014. This major shift in the number of approvals is due to many factors, including the intensified efforts by scientists and clinicians to develop new drugs, especially novel immunotherapies, and changes in the FDA's drug approval process under the leadership of Dr Richard Pazdur.

As immunotherapies redefine the broader cancer treatment paradigm, in breast cancer, CDK 4/6 inhibitors are quietly revolutionizing treatment options for estrogen-receptor-positive metastatic disease. Three exciting drugs - palbociclib, abemaciclib, and ribociclib are in various stages of development.

In February 2015, the agency approved palbociclib in combination with letrozole for treatment of first-line ER-positive metastatic breast cancer, based on results from the PALOMA II trial, which showed a doubling of progression-free survival with the combination. ${ }^{1}$ So far, no drug has shown such an improvement in progression-free survival in hormone-receptor-positive metastatic breast cancer. A year later, in March 2016, the agency approved the combination of palbocicilb with fulvestrant based on PALOMA III results $^{2}$ as a second-line therapy in women with HR-positive, HER2-negative metastatic breast cancer.

In October 2015, another CDK 4/6 inhibitor, abemaciclib, received Breakthrough Therapy Designation from the FDA for use in patients with refractory HR-positive advanced or metastatic breast cancer. Data from the breast cancer cohort expansion of the phase 1 JPBA trial formed the basis of the designation. ${ }^{3}$ The trial focused on the efficacy and safety of abemaciclib in women with advanced or metastatic breast cancer who had received a median of 7 previous systemic treatments. Even in heavily pretreated patients, abemaciclib showed activity as a single agent.

Most recently, in August 2016, the agency gave another breakthrough designation to ribociclib, or LEE011, as a first-line treatment for HR-positive, HER2-negative,

advanced breast cancer based on findings in the phase 3 MONALEESA-2 trial in which ribociclib in combination with letrozole in postmenopausal women who had received no previous therapy for their advanced disease. ${ }^{4}$ Cellular proliferation, mediated by dysregulation of the cell cycle and activation of cyclin-dependent kinases (CDKs) to enhance cell-cycle progression, is the cornerstone of cancer progression. The new generation of selective CDK 4/6 inhibitors, including palbociclib, abemaciclib, and ribociclib, have shown that the inhibitors have an important role in the G1-toS-phase cell-cycle transition, showing improved effectiveness with fewer adverse effects. ${ }^{5}$ The most common side effects are neutropenia, leukopenia, and fatigue. It is worth noting that the incidence of fever neutropenia is extremely rare (less than $2 \%$ ).

Unlike with palbociclib and ribociclib, fatigue was the dose-limiting toxicity of abemaciclib. The side effects for abemaciclib included fatigue (all grades, $41 \%$; grade $3,3 \%)$, diarrhea ( $63 \%$ and $5 \%$, respectively), nausea ( $45 \%$ and $2 \%)$, vomiting ( $25 \%$ and $1 \%$ ), and anorexia ( $17 \%$ and none). Diarrhea was common but manageable with antidiarrheal agents or dose reduction. Hematologic side effects for abemaciclib were less common than they were for palbociclib and ribociclib. ${ }^{6}$

I have learned a number of lessons by using CDK 4/6 inhibitors. Based on data and our experience with patients with metastatic receptor-positive breast cancer, even if they have visceral disease, we can safely start them on the palbociclib-letrozole combination. This will spare a large number of patients from the side effects of chemotherapy. This is a paradigm shift in our decision making. I have also learned that we have to be very patient with palbociclib. We are not going to see a major response right away. We will see reduction in the tumor size, but it may not be as much as we might see with other targeted therapies or chemotherapy agents. We will also see large number of patients achieve stable disease, and a large number of partial responses. As such, we have to educate our patients about the importance of staying on the treatment.

As far as side effects are concerned, we will see a reduction in white blood cells and neutrophils. Follow the pack- 


\section{From the Editor}

age insert for dose modifications. But even with low whitecell counts, patients will do fine and they are not going to get an infection. The incidence of neutropenic fever with palbociclib is very rare (less than $2 \%$ ). It took some time for me to get comfortable with this and to not stop, delay, or dose reduce for uncomplicated neutropenia.

\section{References}

1. Finn RS, Crown JP, Lang I, et al. The cyclin-dependent kinase $4 / 6$ inhibitor palbociclib in combination with letrozole versus letrozole alone as first-line treatment of oestrogen receptor-positive, HER2negative, advanced breast cancer (PALOMA-1/TRIO-18): a randomised phase 2 study. Lancet Oncol. 2015;16(1):25-35.

2. Cristofanilli M, Turner NC, Bondarenko I, et al. Fulvestrant plus palbociclib versus fulvestrant plus placebo for treatment of hormonereceptor-positive, HER2-negative metastatic breast cancer that progressed on previous endocrine therapy (PALOMA-3): final analysis of the multicentre, double-blind, phase 3 randomised controlled trial. Lancet Oncol. 2016;17(4):425-39.

3. Dickler MN, Tolaney SM, Rugo HS, et al. MONARCH1: Results from a phase II study of abemaciclib, a CDK4 and CDK6 inhibitor,
Data from all CDK 4/6 inhibitors are very promising, and these agents will offer highly viable options for our patients with metastatic breast cancer. Looking forward, ongoing studies are now examining the role of palbociclib in earlystage breast cancer (PENELOPE-B, NCT01864746; and PALLAS, NCT02513394).

as monotherapy, in patients with $\mathrm{HR}+/ \mathrm{HER} 2-$ breast cancer, after chemotherapy for advanced disease. J Clin Oncol. 2016;34(suppl; abstr 510).

4. Hortobagyi GN, Stemmer SM Burris HA, et al. Ribociclib as firstline therapy for HR-positive, advanced breast cancer. http://www. nejm.org/doi/full/10.1056/NEJMoa1609709\#t=article. Published October 8, 2016. Accessed October 24, 2016.

5. Finn RS, Aleshin A, Slamon DJ. Targeting the cyclin-dependent kinases (CDK) 4/6 in estrogen receptor-positive breast cancers. Breast Cancer Res. 2016;18(1):17.

6. O'Sullivan CC. CDK4/6 inhibitors for the treatment of advanced hormone receptor positive breast cancer and beyond: 2016 update. Expert Opin Pharmacother. 2016;17(12):1657-67. 